\title{
Contribution à l'étude géophysique d'une nappe profonde dans la région de Gabes (Sud Tunisien)
}

\author{
Contribution to the geophysical study of a deep water table \\ in the Gabes area (South of Tunisia)
}

\author{
par M. Gasmi*, A. Henchiri*, R. Ghoudi**, A. Ben Ayed* et M. Ben Salem* \\ *Ecole Nationale d'Ingénieurs de Gabès \\ ** CRDA-AREE, Gabès, Tunisie.
}

The area of study, located in the northern part of Gabes (South of Tunisia), remains other slightly unknown as far as its deep water table is concerned. The sole existing witness is the 'El Hicha' drilling, dug more than 20 years ago, reaching the water table having a salinity of $5.6 \mathrm{~g} \ell$ at a depth exceeding $100 \mathrm{~m}$. On the other hand, $15 \mathrm{~km}$ North West of this point, another drilling 'Sbih' has reached a water table, with a salinity of $17 \mathrm{~g} /$ at a depth exceeding $150 \mathrm{~m}$.

A geophysics study undertaken by VES is deemed necessary in order to know the limits of this deep water table at 'El Hicha' area. To reach this objective, a measuring study was done in collaboration with CRDA-Gabes. Results have allowed to locate the deep water table in three suitable zones to plant new drilling for exploration.

\section{INTRODUCTION}

L'état actuel des réserves d'eau de la nappe superficielle dans la région "El Ajaira-El Hicha" à trente kilomètres au Nord de Gabès (Sud Tunisien) se trouve insuffisant pour y subvenir aux besoins croissants de l'agriculture. Les informations acquises de l'inventaire des puits non profonds demeurent peu prometteuses à cause des irrégularités du niveau pièzométrique et de la salinité de la nappe superficielle. Cela a suscité le creusement d'un forage profond "Sbih" dans l'espoir de capter la nappe profonde de salinité de l'ordre de $6 \mathrm{~g} / \mathrm{l}$ déjà exploitée depuis longtemps au niveau du forage "El Hicha" à une quinzaine de kilomètres à l'Est de ce point. Malheureusement l'eau produite par le nouveau forage n'est pas exploitable à cause de sa forte salinité (plus de $16 \mathrm{~g} /$ ) et par conséquent il ne s'agit pas de la même nappe.

C'est dans ce contexte que s'intègre le présent travail, dont le but principal est de délimiter la nappe profonde d'El Hicha, tant latéralement que verticalement. Pour ce faire, on a été amené à réaliser une campagne géophysique par Sondages Electriques Verticaux (SEV).

\section{II — SITUATION GÉOGRAPHIQUE ET GÉOLOGIQUE}

La région d'El Ajaira - El Hicha est située dans la zone des basses steppes entre les méridiens $37^{\mathrm{G}} 90^{\prime}$ et $38^{\mathrm{G}} 10^{\prime}$. Elle couvre une superficie de l'ordre de $75 \mathrm{~km}^{2}$.
C'est une zone caractérisée par un paysage monotone de faible gradient altitudinal. Les terrains y affleurant vont du Secondaire au Quaternaire $[1,2]$. Il y a lieu de distinguer : a) le Secondaire représenté uniquement par le Crétacé où on peut noter de bas en haut :

* Crétacé inférieur :

- le Wealdien : affleure uniquement à la terminaison Est de l'anticlinal de Zemlet El Beida (Sud de la zone étudiée). Il est constitué d'argiles gypseuses et d'anhydrite de puissance de l'ordre de $200 \mathrm{~m}$. Il forme le 'Continental Intercalaire'.

- l'Albo-Aptien : visible aux alentours de Zemlet El Beida sur une épaisseur de 20 à $40 \mathrm{~m}$. Il est constitué par une alternance de lits de dolomite, de calcaire dolomitique et de marne argilo-gypseuse.

* Crétacé supérieur :

- le Cénomanien : représenté par un ensemble gypseux passant vers le haut à une alternance de gypse, d'argile et de marne.

- le Turonien : affleure au Zemlet El Beida, au Djebel Dissa et Djebel Rommana (limites Sud-Ouest de la zone d'étude) et formé globalement de dolomites.

b) le Tertiaire : représenté par des séries attribuées au MioPliocène et constituées essentiellement d'argiles gypseuses rougeâtres d'épaisseur très variable, et de sable (de $50 \mathrm{~m}$ d'épaisseur) qui constitue l'aquifère de la nappe profonde de la région. Ces dépôts Mio-Pliocène constituent le substratum des formations quaternaires.

c) le Quaternaire : représenté essentiellement par les trois terrasses emboîtées suivantes : 


\begin{tabular}{|c|c|c|c|c|c|c|c|}
\hline $\begin{array}{l}\mathrm{N}^{\circ} \mathrm{du} \\
\text { puits }\end{array}$ & $\begin{array}{l}\text { Altitude } \\
(\mathrm{m})\end{array}$ & $\begin{array}{l}\text { Niveau } \\
\text { Statique } \\
\text { (m) }\end{array}$ & $\begin{array}{l}\text { Résidu } \\
\operatorname{Sec}(g / 1)\end{array}$ & $\begin{array}{l}\mathrm{N}^{\circ} \mathrm{du} \\
\text { puits }\end{array}$ & $\begin{array}{l}\text { Altitude } \\
(\mathrm{m})\end{array}$ & $\begin{array}{l}\text { Niveau } \\
\text { Statique } \\
\text { (m) }\end{array}$ & $\begin{array}{l}\text { Résidu } \\
\operatorname{Sec}(g / l)\end{array}$ \\
\hline 1 & 17,50 & 7,50 & 5,10 & 20 & 58,00 & 21,80 & 8,54 \\
\hline 2 & 21,50 & 7,70 & 5,30 & 21 & 18,00 & 1,50 & 5,50 \\
\hline 3 & 23,70 & 12,20 & 5,70 & 22 & 12,00 & 2,50 & 5,30 \\
\hline 4 & 15,00 & 5,00 & 5,45 & 23 & 22,00 & 9,70 & 8,31 \\
\hline 5 & 23,50 & 11,00 & 5,45 & 24 & 18,00 & 6,00 & 7,26 \\
\hline 6 & 14,00 & 0,00 & 5,20 & 25 & 20,50 & 9,20 & 11,50 \\
\hline 7 & 18,00 & 0,00 & 5,20 & 26 & 24,00 & 13,40 & 10,40 \\
\hline 8 & 23,90 & 4,90 & 5,04 & 27 & 6,00 & 4,00 & 4,52 \\
\hline 9 & 28,80 & 10,80 & 5,40 & 28 & 10,00 & 6,00 & 5,00 \\
\hline 10 & 24,00 & 7,00 & 5,90 & 29 & 14,00 & 0,00 & 5,26 \\
\hline 11 & 21,00 & \multicolumn{2}{|c|}{ sec à $18,00 \mathrm{~m}$} & 30 & 8,00 & 3,80 & 5,64 \\
\hline 12 & 21,00 & 21,00 & 16,50 & 31 & 26,00 & 17,60 & 5,50 \\
\hline 13 & 33,00 & 5,10 & 11,00 & 32 & 23,00 & 12,20 & 5,70 \\
\hline 14 & 39,00 & \multicolumn{2}{|c|}{$\sec$ à $14,00 \mathrm{~m}$} & 33 & 20,50 & - & 5,90 \\
\hline 15 & 39,50 & 8,00 & 9,90 & 34 & 20,00 & - & 6,90 \\
\hline 16 & 49,00 & 14,50 & 8,94 & 35 & 18,00 & - & 7,60 \\
\hline 17 & 51,00 & \multicolumn{2}{|c|}{$\mathrm{sec}$ à $15,00 \mathrm{~m}$} & 36 & 24,00 & - & 6,90 \\
\hline 18 & 58,00 & \multirow{2}{*}{\multicolumn{2}{|c|}{$\frac{\mathrm{sec} \text { à } 9,70 \mathrm{~m}}{\mathrm{sec} \text { à } 13,00 \mathrm{~m}}$}} & 37 & 29,00 & 16,80 & 6,80 \\
\hline 19 & 61,00 & & & & & & \\
\hline
\end{tabular}

Tableau 1: Résultats de l'inventaire des puits superficiels d'El Ajaira (AREE-CRDA-Gabès, décembre 1995).
* la haute terrasse : emboîtée dans le glacis Pleistocène et constituée d'un matériel grossier d'épaisseur maximale de $5 \mathrm{~m}$ renfermant des lentilles de limons rouges et beiges à concrétions calcaires. Cette terrasse est attribuée au Pleistocène moyen [2].

* la terrasse moyenne : emboîtée dans des niveaux plus anciens et constituée par des limons rouges à jaunes et d'argile, renfermant des lentilles de sable grossier et de galets. L'ensemble est coiffé d'une croûte gypseuse. Cette terrasse correspond à la formation 'El Akarit' et est d'âge Pleistocène supérieur à Halocène inférieur [2].

* la basse terrasse : définie par des dépôts de galets et de graviers d'épaisseur maximale de $2 \mathrm{~m}$ (Fig. 1).

\section{III — CADRE HYDROGÉOLOGIQUE}

En évoquant l'hydrogéologie de la région étudiée, il faut parler successivement des deux nappes existantes : superficielle et profonde.

\subsection{Nappe superficielle}

L'inventaire des puits superficiels qui consiste à mesurer le niveau du plan d'eau et à calculer le résidu sec par analyses chimiques a été réalisé en décembre 1995 par l'Arrondissement des Ressources en Eau (AREE-CRDA de Gabès). Cet inventaire a concerné 37 puits de profondeur allant jusqu'à $70 \mathrm{~m}$ (tableau 1).

En fait, la nappe phréatique superficielle se trouve dans deux niveaux différents $[3,4]$ à savoir :

* premier niveau : capté par la majorité des puits de profondeur inférieure à $50 \mathrm{~m}$. Il est formé par des séries sablo-argileuses riches en gypse, ce qui contribue à augmenter le résidu sec jusqu'à $7 \mathrm{~g} / \mathrm{l}$.

* deuxième niveau : se trouve à une profondeur comprise entre 50 et $70 \mathrm{~m}$ et formé par des séries sableuses pauvres en gypse, le résidu sec étant de 5 à $6 \mathrm{~g} / \mathrm{l}$.

\subsubsection{Piézométrie}

En l'absence d'une altimétrie pour tous les puits inventoriés, on s'est basé sur la carte topographique pour en déduire les altitudes des puits manquants. La carte piézométrique (Fig.2) montre que l'écoulement souterrain est globalement vers l'Est, c'est-à-dire vers la mer qui constitue l'exutoire naturel de cette nappe superficielle.

\subsubsection{Salinité}

La carte (Fig.3) montre que la salinité de la nappe est très variable d'un endroit à un autre et varie de 4,5 à $16,5 \mathrm{~g} / \mathrm{l}$. La zonalité de salinité peut s'expliquer soit par la présence locale de gypse, soit par une alimentation profonde en eau très chargée. D'autre part en examinant ensemble les deux cartes (Fig. 2 et Fig.3), on peut prétendre qu'on a affaire à deux nappes différentes, sinon comment peut-on expliquer qu'un puits de $11 \mathrm{~g} / 1$ situé à l'amont puisse alimenter un autre de $5 \mathrm{~g} / \mathrm{l}$ à l'aval ?

Enfin, on peut conclure que les ressources de la nappe superficielle de la région El Ajaira-El Hicha sont assez limitées et généralement chargées, ce qui a conduit à chercher des potentialités plus profondes, autrement dit la nappe profonde.

\subsection{Nappe profonde}

Elle est captée par le puits 'El Hicha' n6044. L'aquifère est constitué par une couche sableuse d'âge mio-pliocène, d'épaisseur $60 \mathrm{~m}$ et de profondeur variant de 100 à $180 \mathrm{~m}$. Son résidu sec est de 5,6 g/l. D'après les études géologiques, cette nappe constitue l'extension vers le Nord de celle de 'Djeffara' qui est bien connue au Sud tunisien. On pense aussi que cette nappe est en communication hydraulique avec les aquifères : le 'Complexe Terminal' capté dans des calcaires cénomarniens dans la région de Gabès et le 'Continental Intercalaire', capté dans des sables Wealdiens dans le secteur de 'Draa Ouedref' à l'Ouest de Gabès. 


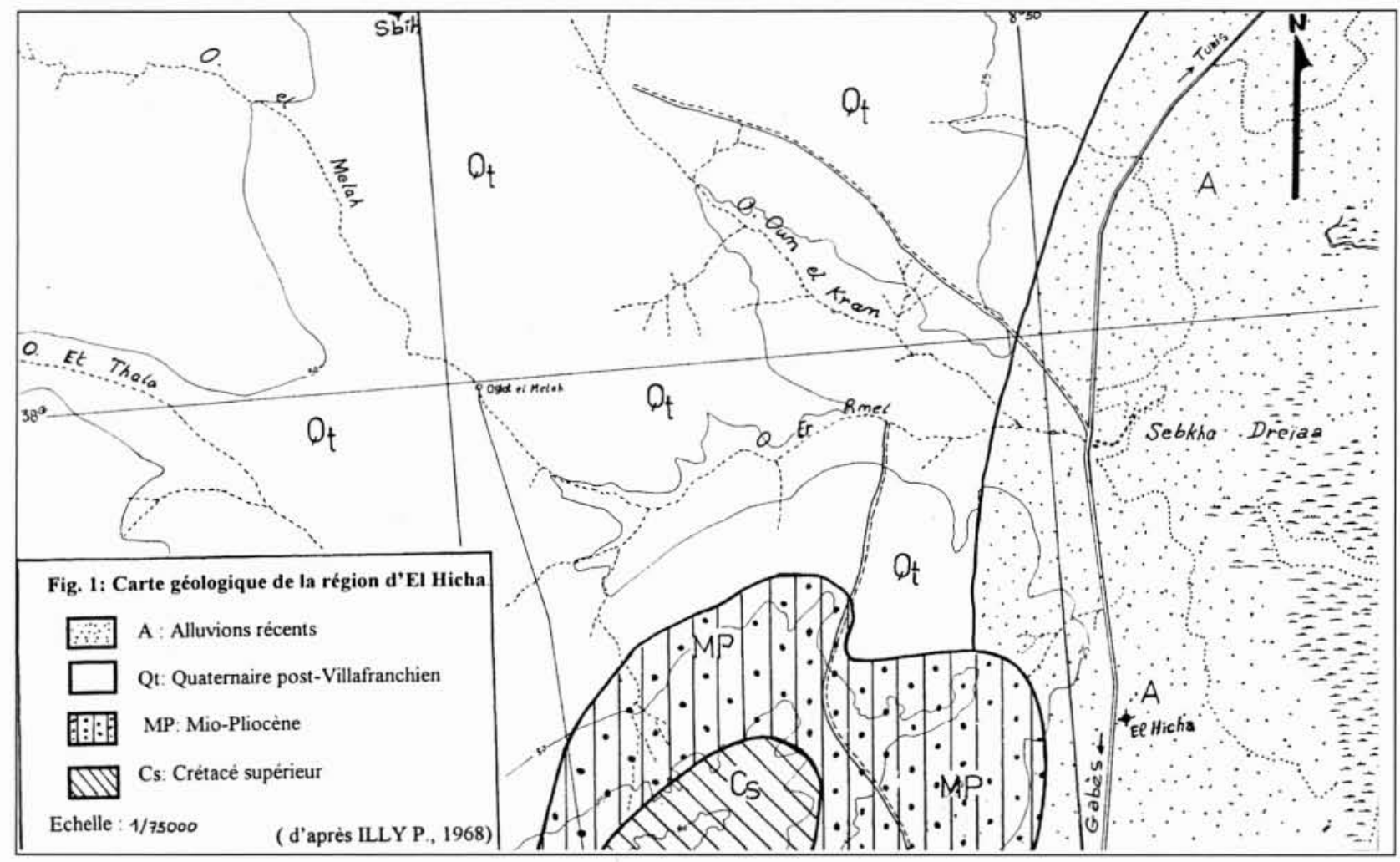

1. Carte géologique de la région d'El Hicha

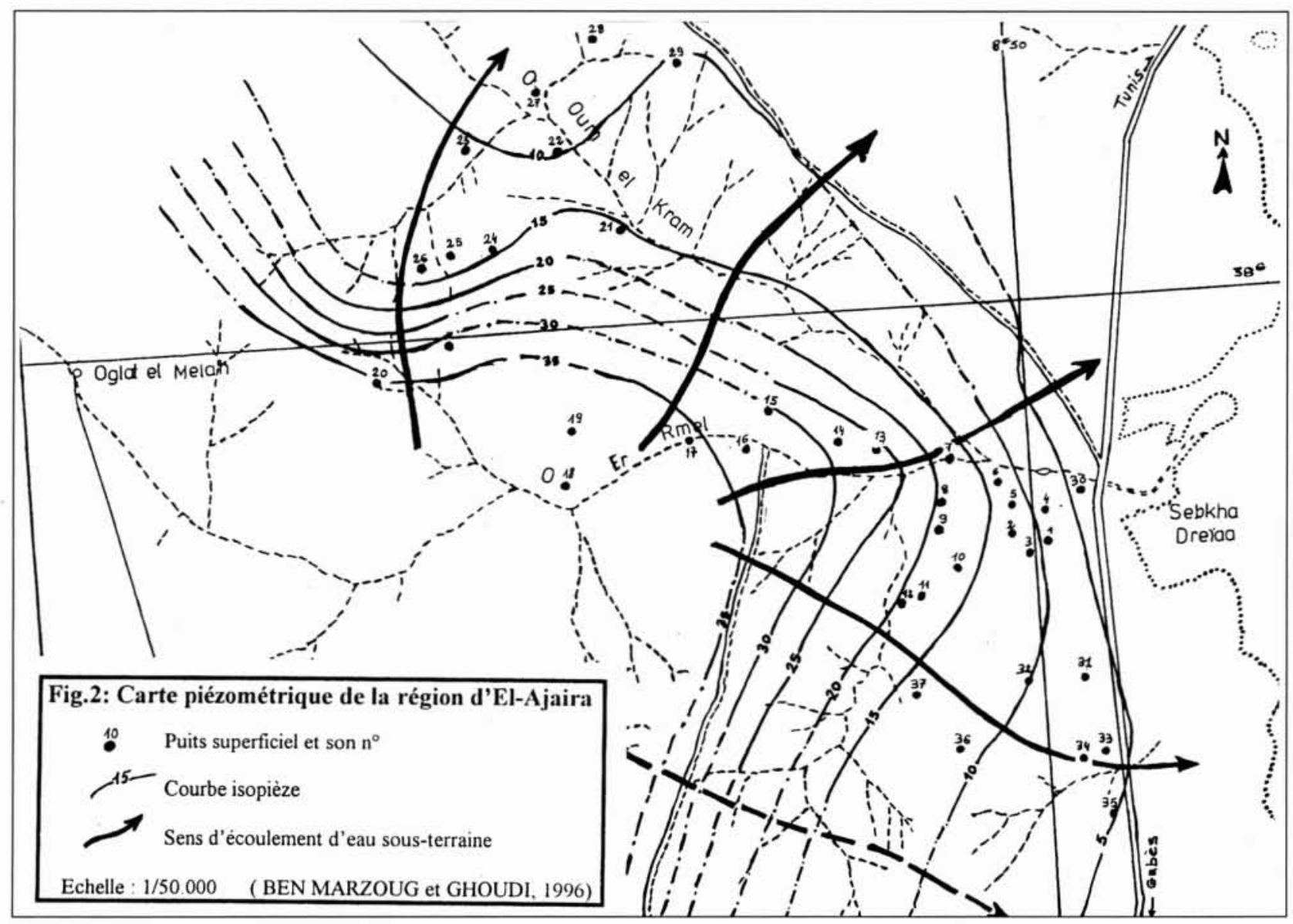

2. Carte piézométrique de la région d'El-Ajaira 


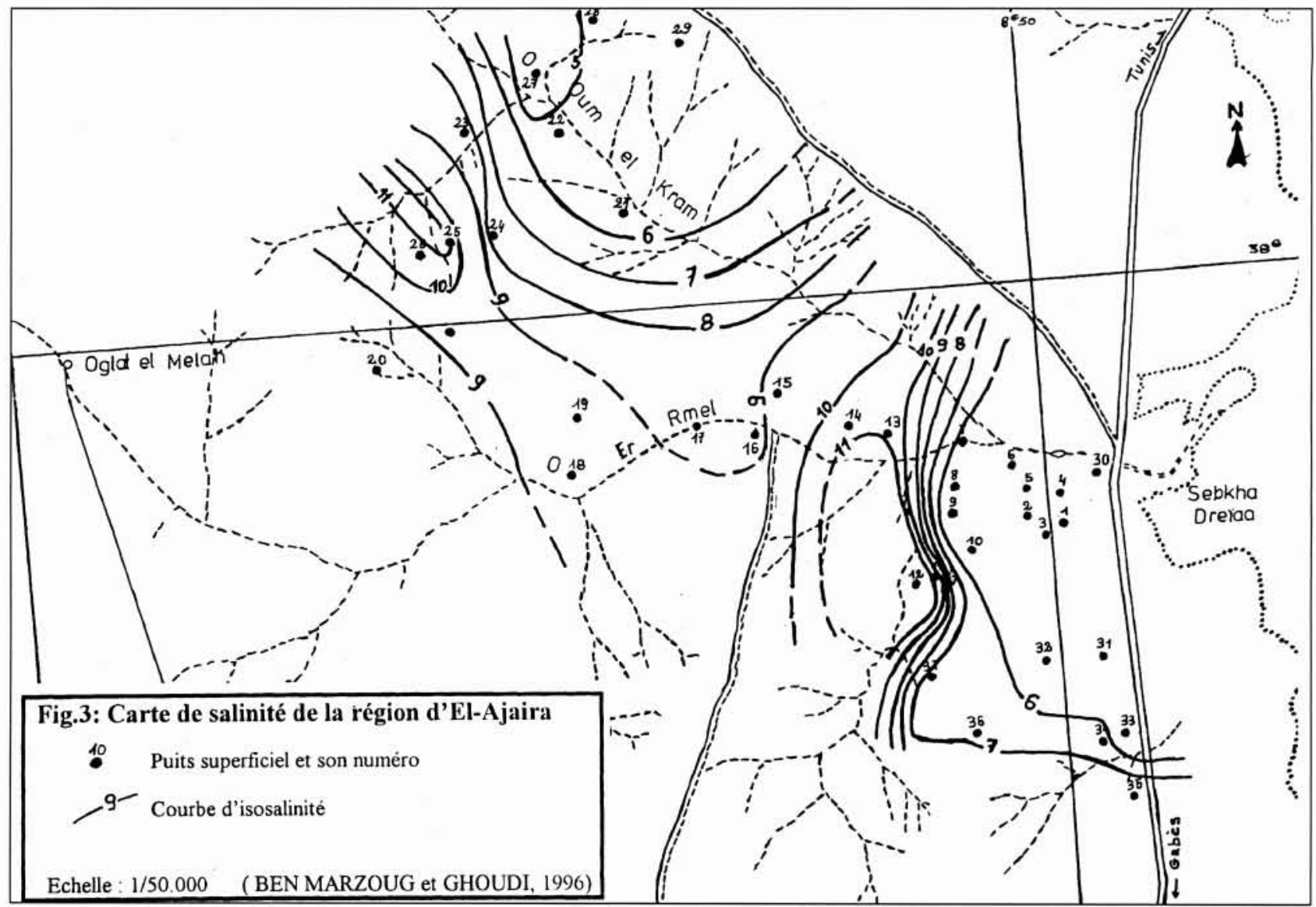

3. Carte de salinité de la région d'El-Ajaira

La reconnaissance de cette nappe passe par une étude géophysique qui permet de mieux comprendre la structure du sous-sol grâce à l'identification des résistivités et des épaisseurs des couches souterraines.

\section{IV —ÉTUDE GÉOPHYSIQUE}

\subsection{Aperçu théorique}

La méthode géophysique jugée la mieux adaptée à la région d'étude est le Sondage Electrique Vertical (SEV). Elle se base sur la mesure de la résistivité des couches qui varie d'une roche à une autre et dépend de plusieurs facteurs. La présence de l'eau (teneur et salinité) en est parmi les principaux.

En pratique, on utilise un quadripôle AMNB : par deux électrodes $\mathrm{A}$ et $\mathrm{B}$ on injecte un courant continu d'intensité $\mathrm{I}$, et entre les deux autres $\mathrm{M}$ et $\mathrm{N}$ on mesure la différence de potentiel $\Delta \mathrm{V}=\mathrm{V}_{\mathrm{M}}-\mathrm{V}_{\mathrm{N}}$. Pour un terrain homogène et isotrope de résistivité réelle $\rho$, on a :

$$
\Delta \mathrm{V}=\frac{\rho \mathrm{I}}{2 \pi}\left[\frac{1}{\mathrm{MA}}-\frac{1}{\mathrm{NA}}-\frac{1}{\mathrm{MB}}+\frac{1}{\mathrm{NB}}\right] \text {. }
$$

On peut généraliser cette formule à un autre terrain non homogène et en déduire sa résistivité apparente $\rho_{\mathrm{a}}$, telle que $\rho_{\mathrm{a}}=\mathrm{K}(\Delta \mathrm{V} / \mathrm{I}(\Omega \cdot \mathrm{m})$ avec :

- K : coefficient géométrique du dispositif utilisé,

- $\Delta \mathrm{V}$ : potentiel mesuré entre les électrodes $\mathrm{M}$ et $\mathrm{N}(\mathrm{mV})$,

- I : intensité du courant injecté entre les électrodes A et B $(\mathrm{mA})$.
L'interprétation des données d'un SEV consiste à trouver le nombre, les résistivités réelles et les épaisseurs des couches du terrain au point prospecté (dans la limite de la profondeur d'investigation de la méthode).

\subsection{Application à la nappe d'El Ajaira-El Hicha}

Le matériel utilisé est un résistivimètre de type Syscal-R2 (construction IRIS-Instruments - France). Le dispositif adopté est celui de Schlumberger. La campagne a totalisé vingt-huit SEV (dix-sept SEV sont repris de la campagne de Clary 1969 et réinterprétés) répartis suivant trois profils (Fig.4) tels que :

- profil P1 : de direction Nord-Sud comprend 11 SEV,

- profil P2 : de direction Est-Ouest comprend 6 SEV,

- et profil P3 : de direction Nord Ouest-Sud Est comprend 11 SEV.

L'interprétation des diagrammes $\rho_{\mathrm{a}}=\mathrm{f}(\mathrm{AB} / 2)$ est améliorée à l'ordinateur à l'aide du logiciel PISE4 [5]. Les résultats de quelques SEV sont présentés sur la figure 5 pour comparaison.

\subsubsection{Interprétation qualitative (pseudosections)}

La réalisation d'une pseudosection consiste à placer sur un papier semi-logarithmique sous tous les SEV (suivant le profil considéré) les valeurs des résistivités apparentes correspondant aux longueurs $\mathrm{AB} / 2$, puis à tracer les courbes d'isorésistivité. Ce procédé permet de fournir une image qualitative sur la structure du sous-sol suivant le profil considéré en mettant en évidence les différentes anomalies conductrices et résistantes ainsi que les relations entre elles. 


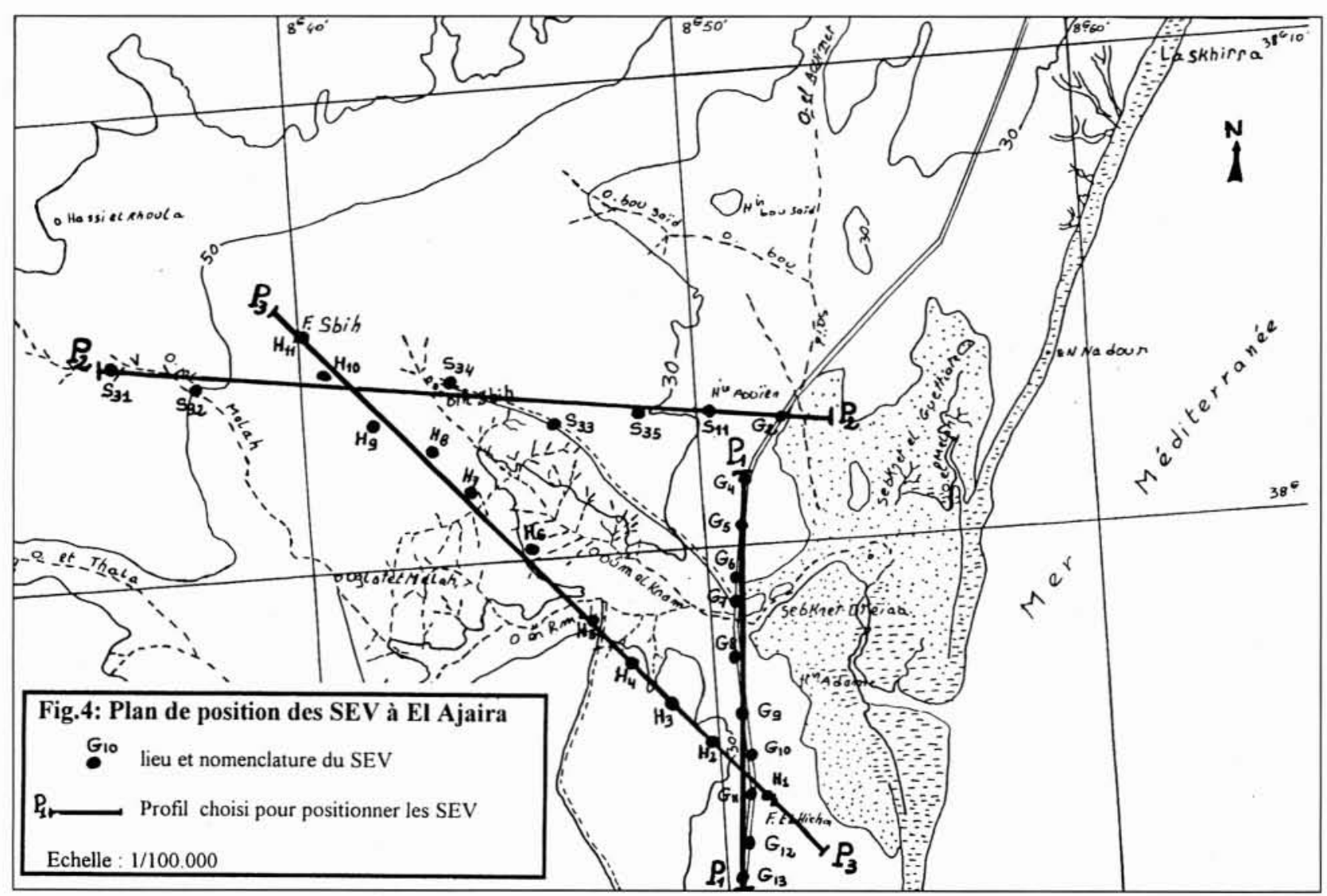

4. Plan de position des SEV à El Ajaira

- profil P1 (Fig.6) : présente deux anomalies résistantes superficielles, la première localisée sous les SEV G4, G5 et G6, la deuxième sous les SEV G8, G9, G10, G11 et G12. En allant vers le bas (en profondeur) les terrains deviennent plus conducteurs avec une résistivité intermédiaire de l'ordre de $5 \Omega$.m et continue sur presque tout le profil. Vers le bas il y a réapparition de deux anomalies relativement résistantes ( $8 \Omega . \mathrm{m})$ présentant la couche qui renferme la nappe profonde.

- profil P2 (Fig.7) : suivant cette pseudosection les couches sont tabulaires avec une tendance d'approfondissement en dessous du SEV S31. On retrouve presque les mêmes couches géoélectriques que le profil précédent, sans toucher le dernier résistant.

- profil P3 (Fig.8) : on a le même cas de figure que le profil $\mathrm{P} 1$ : les corps résistants superficiels, le conducteur du milieu et le résistant d'en bas présentant l'aquifère de la nappe profonde sous les SEV H4, H5 et H6.
4.2.2 Interprétation Quantitative (coupes géoélectriques) : suivant le profil topographique considéré on place les lieux des SEV et sous chaque point on reporte les résistivités réelles en fonction des épaisseurs déduites de l'interprétation des courbes de $\rho_{a}=f(A B / 2)$. Puis on relie par interpolation les niveaux de même résistivité, ce qui donne une sorte de coupe géologique. Mais comme les couches sont définies par un paramètre physique, on l'appelle coupe géoélectrique.

Pour mieux connaître les caractéristiques électriques de l'aquifere recherché, on a réalisé le SEV H1 à côté du forage 'El Hicha' pour servir d'étalon et en généraliser ses résultats aux autres SEV. La comparaison des résultats de ce SEV avec ceux du forage sont présentés dans le tableau 2.

On constate que l'aquifere profond aurait une résistivité réelle supérieure à $8 \Omega$.m et serait composé de sable et de gravier de Quartz. Il est surmonté d'une épaisse couche argileuse de résistivité comprise entre 3 et $5 \Omega$.m. En dessus on trouve les terrains sableux, graveleux et argileux renfermant

\begin{tabular}{|c|c|c|c|c|c|}
\hline \multicolumn{4}{|c|}{ Forage 'El Hicha' } & \multicolumn{2}{|c|}{ SEV HI } \\
\hline \multirow{2}{*}{$\begin{array}{l}\text { épaisseur } \\
\text { (m) }\end{array}$} & \multicolumn{2}{|c|}{ Diagraphie } & \multirow{2}{*}{$\begin{array}{c}\text { nature lithologique des } \\
\text { terrains traversés }\end{array}$} & \multirow{2}{*}{$\begin{array}{l}\text { résistivité } \\
(\Omega \mathrm{m})\end{array}$} & \multirow{2}{*}{$\begin{array}{l}\text { épaisseur } \\
\text { (m) }\end{array}$} \\
\hline & $\begin{array}{c}\text { PS } \\
(\mathrm{mV})\end{array}$ & $\begin{array}{c}\mathrm{GN} \\
(\Omega \mathrm{m})\end{array}$ & & & \\
\hline 12 & - & - & $\begin{array}{c}\text { argiles, débris de gypse } \\
\text { et gravier }\end{array}$ & 20 à 30 & 9 \\
\hline 33 & - & - & $\begin{array}{l}\text { sable, gravier et sable } \\
\text { argileux }\end{array}$ & 7 & 30 \\
\hline 66 & +4 & 5 & $\begin{array}{c}\text { argile brune et argile } \\
\text { rouge }\end{array}$ & 3 & 68 \\
\hline 54 & -8 & 8 & $\begin{array}{c}\text { sable et gravier de } \\
\text { Quartz }\end{array}$ & 10 & - \\
\hline
\end{tabular}

Tableau 2: Comparaison des résultats du SEV HI avec ceux du forage 'El Hicha' 
Eau : ressources, aménagements, environnement
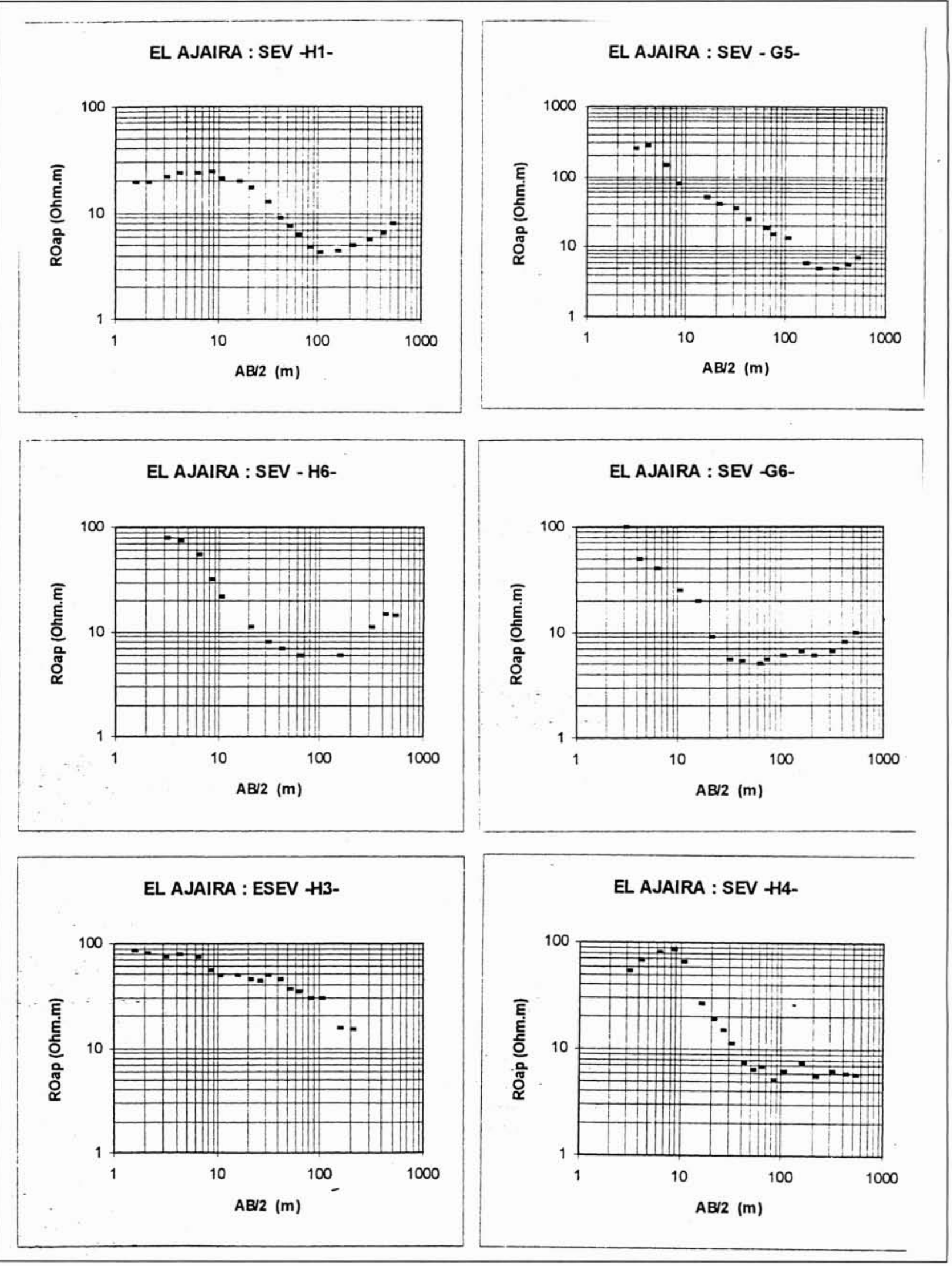

5. Résultats de quelques SEV réalisés à El Ajaira 


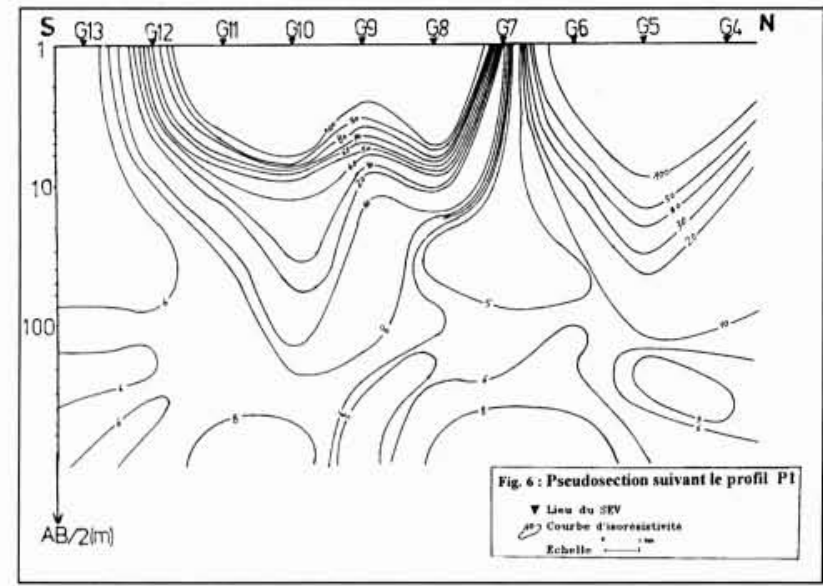

6. Pseudosection suivant le profil P1

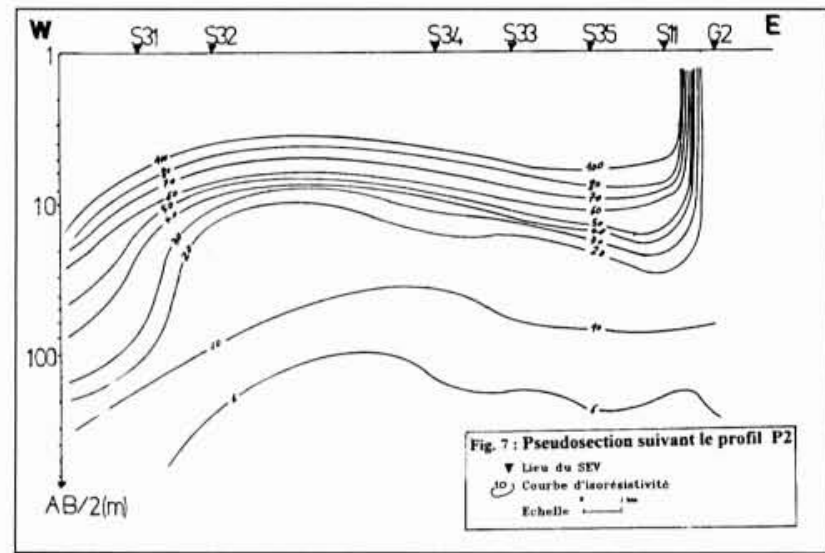

7. Pseudosection suivant le profil P2

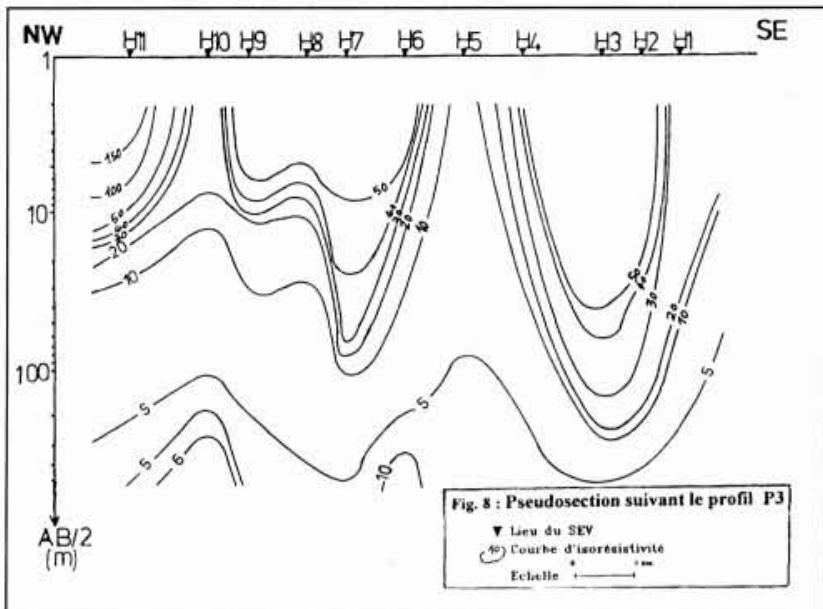

8. Pseudosection suivant le profil P3

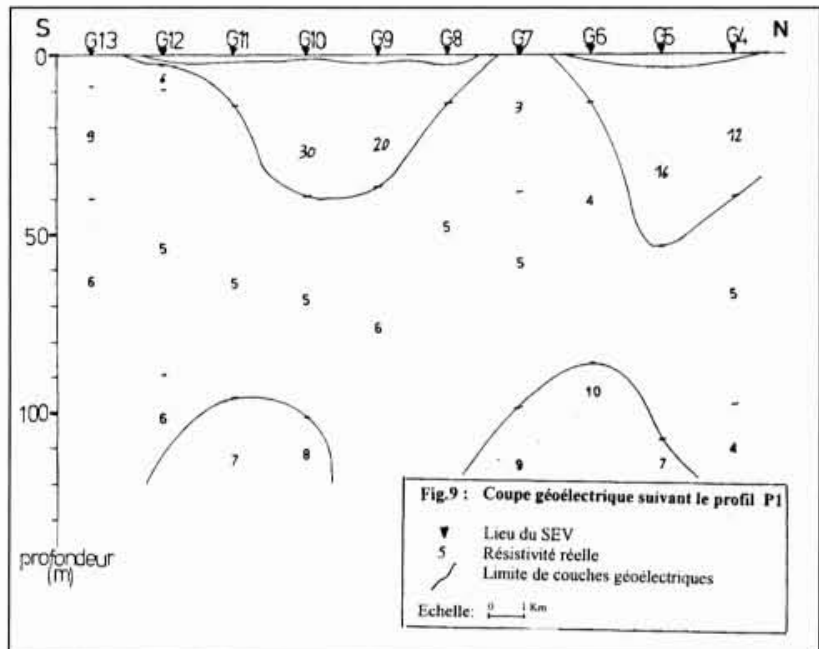

9. Coupe géoélectrique suivant le profil P1

pense aussi que le long de ce profil il n'y a pas une anomalie structurale (faille) faisant basculer la couche aquiferre. La variation de profondeur est probablement due à une géomorphologie irrégulière avant le dépôt de la couche argileuse.

- profil 2 (Fig.10) : le long de ce profil et de haut en bas, on assiste à la présence continue et régulière des couches suivantes :

* une couche résistante qui constitue le mélange d'en haut, *une couche relativement résistante $(\rho \geq 10 \Omega$.m) qui représente la nappe phréatique superficielle,

* une dernière couche conductrice représentant les argiles.

La couche renfermant l'aquifère profond n'est pas présente : elle est probablement à une profondeur plus importante que celle d'investigation de la méthode avec $\mathrm{AB} /=300 \mathrm{~m}$.

Dans la partie Ouest du profil et à partir du SEV S32, on assiste à un approfondissement de tous les terrains présents.

- Profil P3 (Fig.11) : c'est le profil présentant les résultats des mesures réalisées au cours de la dernière campagne. De haut en bas on a les couches suivantes :

- Une couche résistante et continue le long du profil présentant le mélange d'en haut. Comme pour le profil P1, on a deux creux, le premier sous les SEV H2, H3 et $\mathrm{H} 4$ et le deuxième sous les SEV H7 et H8. Ces deux creux représentent les anciens cours des deux oueds : Er Rmel et Oum El Kram. 


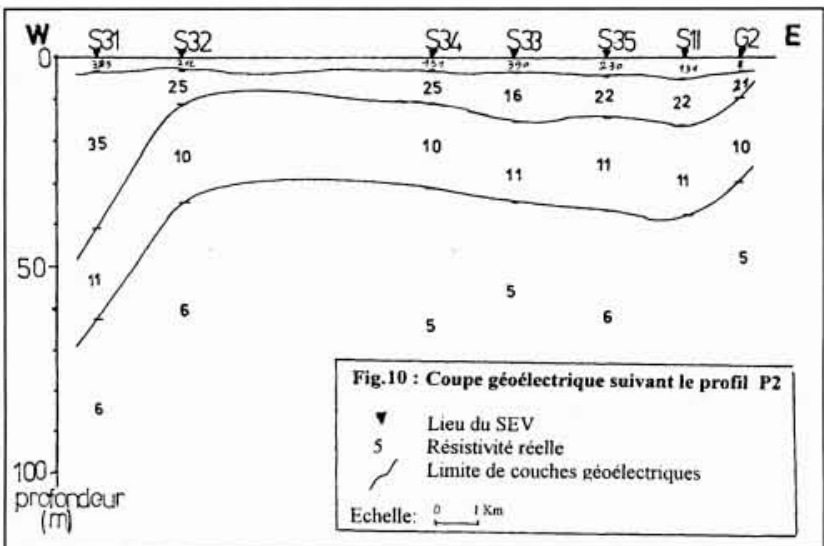

10. Coupe géoélectrique suivant le profil $\mathbf{P 2}$

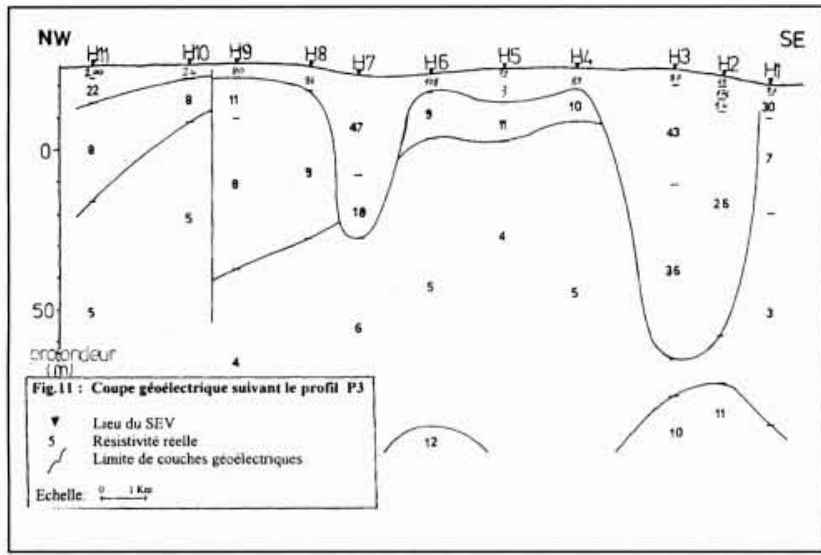

11. Coupe géoélectrique suivant le profil P3

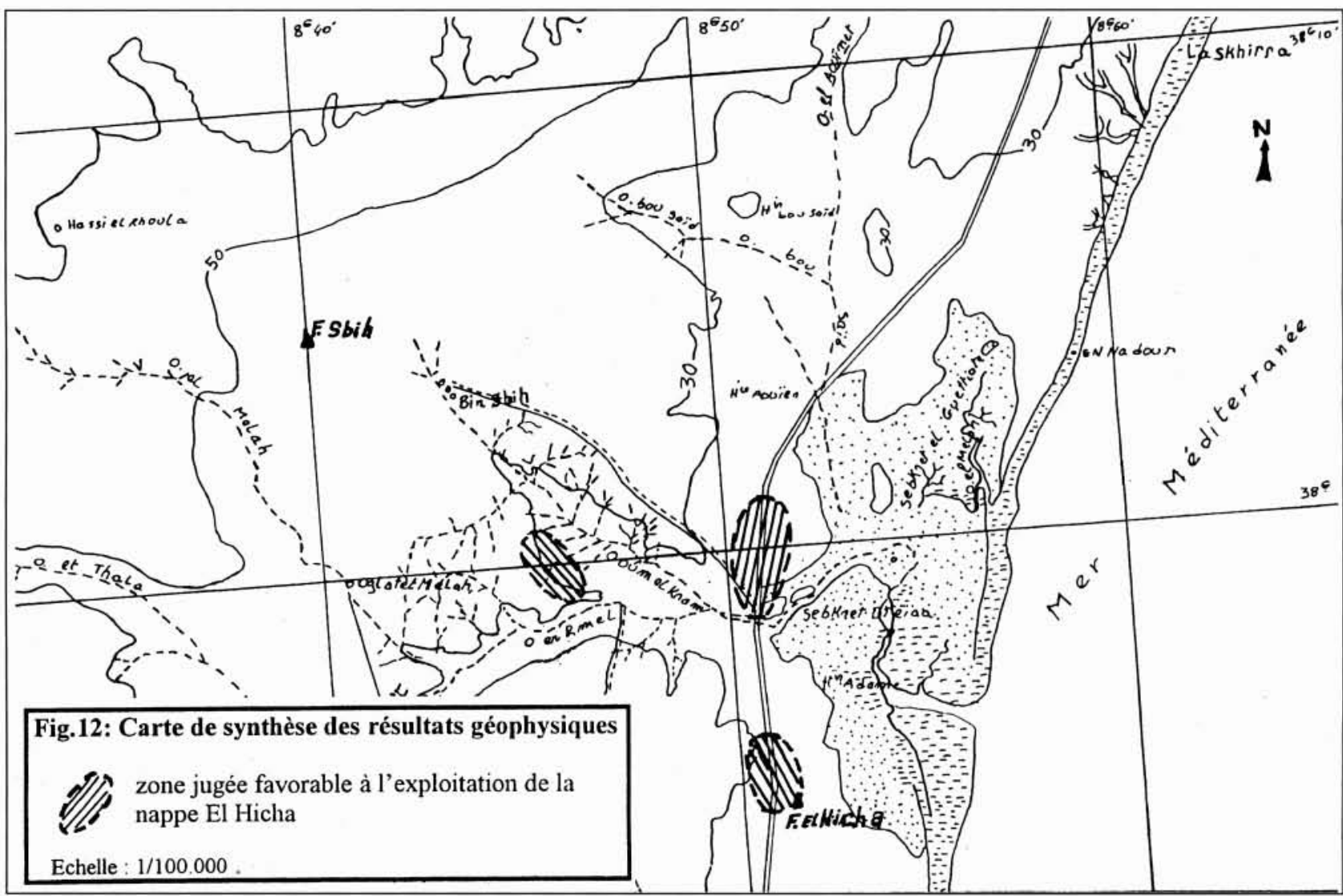

12. Carte de synthèse des résultats géophysiques.

- Une deuxième couche relativement résistante, représentant l'aquifère de la nappe superficielle. Cette couche a un pendage vers le Nord Ouest où elle s'épaissit. Elle présente deux phénomènes anormaux : d'abord elle est entaillée par le remplissage des deux anciens creux, ce qui explique entre autres les variations du niveau piézométrique et de la salinité de cette nappe superficielle ; ensuite, dans la partie Nord Ouest et entre les SEV H9 et H10, elle est traversée par une faille (probablement verticale) qui a fait basculer vers le haut le compartiment Nord Ouest, et vers le bas le compartiment Sud Est. Cette faille a un rejet supérieur à $50 \mathrm{~m}$.

- Une troisième couche conductrice représentant les argiles. Elle est traversée par la même faille, ce qui peut signifier que cette dernière est profonde et peut même atteindre l'aquifere profond.

- Une quatrième couche relativement résistante $(\rho \geq 10 \Omega$.m) représentant l'aquifère profond. Elle est présente uniquement en deux endroits : d'abord sous les SEV H1, H2 et H3, ensuite sous le SEV H6. En dehors de ces deux endroits et jusqu'à la trace de la faille, cette couche est probablement continue mais plus profonde.

Il est possible que la faille détectée par ce travail mette en contact la nappe profonde de salinité $17 \mathrm{~g} / \mathrm{l}$ captée à 'Sbih' avec la nappe superficielle : c'est ce qui peut expliquer, entre autres, la forte salinité de celle-ci dans cette région ( $\mathrm{cf}: \S 3.1 .2$ ). 


\section{$\mathrm{V} \square$ CONCLUSION}

L'étude géophysique entamée dans la région 'EL Ajaira -El Hicha' au nord de Gabès (Sud Tunisien) a permis de fournir une image du sous-sol de la zone prospectée et des précisions sur sa nappe profonde. Les principaux résultats sont les suivants :

- Les couches reconnues dans la zone sont au nombre de quatre : la première est représentée par une nature lithologique très diversifiée. La deuxième est constituée par des sables et renferme la nappe superficielle. La troisième couche est épaisse et représentée par des argiles. La dernière couche qui renferme la nappe profonde est représentée par des sables.

- La nappe profonde captée par le forage 'El Hicha' a été atteinte en trois zones (Fig.12) jugées favorables à la prospection et l'exploitation (nouveau forage) de l'aquifere profond recherché. En dehors de ces trois zones, il est nécessaire de pousser la prospection géophysique pour s'assurer d'abord de la continuité de cette nappe et ensuite préciser sa profondeur.

- Dans la partie Nord Ouest de la zone prospectée, une faille profonde a été détectée. Elle a probablement mis en contact la nappe profonde captée à 'Sbih' avec la nappe superficielle et par drainage vertical elle a contaminé cette dernière, ce qui peut aider à expliquer sa forte salinité.

Dans l'avenir, il faut pousser l'étude géophysique en multipliant les SEV afin d'avoir plus de précisions sur l'image souterraine. Il est conseillé aussi d'utiliser des lignes de courant allant jusqu'à $\mathrm{AB} / 2=1000 \mathrm{~m}$ ou plus. Cela a pour objectif de voir la relation existante entre la nappe d'El Hicha, de salinité de l'ordre de $6 \mathrm{~g} / \mathrm{l}$, et celle de Sbih, de salinité supérieure à $17 \mathrm{~g} / \mathrm{l}$.
Remerciements: au terme de ce travail, les auteurs remercient vivement messieurs Hamed Ben Dhia (Ecole Nationale d'Ingénieurs de Sfax-Tunisie) et Pierre Andrieux (Université Pierre \& Marie Curie, Paris-6-France) pour leurs conseils.

\section{Références}

[1] ILLY P. (1968), - Région de Sidi Mheddheb, étude hydrogéologique préliminaire. Rapport interne du Bureau de l'Inventaire des Ressources Hydrauliques, Tunis 1968

[2] ZOUARI K. (1988), - Géochimie et sédimentologie des dépôts continentaux d'origine aquatique du quaternaire supérieur du Sud Tunisien. Interprétation par géohydrologiques et par géoclimatiques. Thèse de doctorat d'Etat. Université Paris-Sud, centre d'Orsay, pp: 231, Juin 1988.

[3] NJAH M et GHRAB A. (1979), - Compte rendu de fin des travaux et d'essais de débit de forage El Hicha $\mathrm{n}^{\circ} 18767 / 5$. Rapport interne de la Direction des Ressources en Eau, Tunis 1979.

[4] BEN MARZOUG M. et GHOUDI R. (1996), - Rapport préliminaire sur l'inventaire des puits de la région d'El Ajaira. Rapport interne AREECRDA-Gabès, 1996.

[5] GABALDA G et TABBAGH J. (1994), - Note explicative du Programme d'Interprétation de Sondages Electriques - PISE4- Version 4. ORSTOM - CRG -CNRS, France 1994.

[6] ASTIER J. L. (1971), - Géophysique appliquée à l'hydrogéologie. Edition Masson \& Cie, pp:272, Paris 1971.

[7] CASTANY G. (1982), - Principes et méthodes de l'hydrogéologie. Edition Bordas, Paris, pp : 236, France, 1982.

[8] CLARY P. (1969), - Prospection électrique dans la région de Graiba, Hachichina, Skhira, plateau d'Hamilet, El babouch et Djebel Zamlet El Beida. Rapport interne de la Division des Ressources Hydrauliques, Arrondissement des Sondages, Section des Etudes Géophysiques, Tunis 1969. 